\title{
MyoRing Treatment of Keratoconus
}

\section{Albert Daxer}

\section{ABSTRACT}

A case of bilateral keratoconus after corneal cross-linking in both eyes and ineffective ring segment implantation in the left eye is presented. The ineffective ring segment was removed and instead, a corneal pocket was created and a MyoRing was inserted. In this case study, the currently available options of treatment are presented and the differences between ring segments and MyoRing are discussed in detail.

Keywords: CISIS, ICRS, Keratoconus, MyoRing, Ring segment.

How to cite this article: Daxer A. MyoRing Treatment of Keratoconus. Int J Kerat Ect Cor Dis 2015;4(2):76-83.

\section{Source of support: Nil}

Conflict of interest: The author has a financial interest in DIOPTEX GmbH.

The data have been presented in part at the annual meetings of the ESCRS 2013 (Amsterdam) and 2014 (London) as well as at the Keratoconus Expert Meetings 2012 in Milano and 2013 Amsterdam.

\section{INTRODUCTION}

Keratoconus is a rare disease in Europe and North America, where it occurs in only 1 out of 1000 to 2000 inhabitants. ${ }^{1}$ There are endemic and epidemic global regions, particularly the near and Middle East, where keratoconus occurs much more often with incidence rates in the double-digit percent range. ${ }^{2}$

The aim of this publication, which draws on a relatively common but exemplary case, is to provide an update of current knowledge about keratoconus and outline the existing methods of treatment.

\section{CASE REPORT}

A 27-year-old patient from Switzerland with symptoms of keratoconus in both eyes presented at my medical practice to seek consultation about keratoconus. He had undergone corneal cross-linking in both eyes at a university

Director and Associate Professor

Gutsehen Eye Center, Ybbs, Stauwerkstraße 1; Department of Ophthalmology, University Hospital Innsbruck, Innsbruck Austria

Corresponding Author: Albert Daxer, Director and Associate Professor, Gutsehen Eye Center, Ybbs, Stauwerkstraße 1; Department of Ophthalmology, University Hospital Innsbruck Innsbruck, Austria, e-mail: daxer@gutsehen.at hospital in his home country to halt the progression of his disease, where also intracorneal ring segments (ICRS) had been implanted in the left cornea to rehabilitate his vision. He reported that after ICRS treatment he had consistently been suffering from poor vision, and that his vision in the treated eye had further deteriorated in the course of the first year following the surgical intervention and that he was dissatisfied with the outcome. He wears a contact lens in the right eye, which he feels quite comfortable with. He had problems wearing a contact lens in the left eye, and wanted the ring segments to be removed and a MyoRing (SehRing) implanted in this eye.

The patient presented with the following eye exam findings:

- OD CDVA 1.0 with -5.75 s -1.75 c $\times 170^{\circ}$, UDVA 0.05

- OS CDVA 0.3 with -12.0 s $-7.75 c \times 170^{\circ}$, UDVA 0.2

Corneal topography in the left eye by means of a Pentacam eye scanner (Oculus $\mathrm{GmbH}$, Germany) revealed SIM K1 = 46.9, SIM K2 = 52.2 and a corneal thickness of $447 \mu \mathrm{m}$ at the thinnest spot. The topography measurements are depicted in Figure 1.

Following the patient's justified opinion that his ICRS treatment had been ineffective, I removed the implanted ring segments in my medical practice. To this end, I reopened the access to the circular tunnel through the radial incision, which was already scarred, and pulled out the segments from the circular tunnel through this access. The radial incision in the cornea was then resutured at the 12-o'clock position by using a 10-0 nylon filament. The sutures were removed after 4 months.

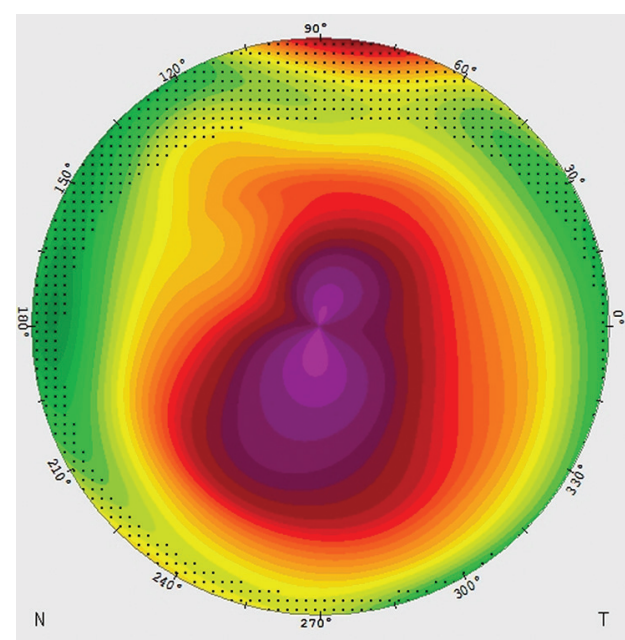

Fig. 1: Sagittal corneal topography of the left eye with ring segments and after cross-linking 
After two more months, MyoRing implantation using local anesthetic eye drops was performed. By using a PocketMaker ultrakeratome (DIOPTEX GmbH, Austria), a lamellar, virtually fully closed corneal pocket (diameter: $9 \mathrm{~mm}$ ) was cut into the cornea at a corneal depth of 300 $\mu \mathrm{m}$. Through a $4 \mathrm{~mm}$ wide temporal lamellar access, a MyoRing (DIOPTEX GmbH, Austria) with a diameter of $6 \mathrm{~mm}$ and a thickness of $300 \mu \mathrm{m}$ was inserted into the corneal pocket. In a next step, the MyoRing was centrally positioned inside the corneal pocket according to the manufacturer's training guidelines by using the intersection point of the optical axis and the corneal surface as a reference. The eye was actively fixating to the surgical microscope by means of a specially equipped lighting and fixing system suited for the purpose.

MyoRing implantation is a surgical intervention that differs significantly from the implantation of ring segments (ICRS).

The internationally patented MyoRing is not a ring segment (ICRS), but a closed full ring implant with two a priori mutually exclusive properties: it is extremely rigid, with a high effective elastic modulus which allows to change the geometry of the cornea, yet flexible (shape memory) so it can be inserted through a tiny lamellar opening in the cornea in a biomechanically neutral manner.

As will be discussed in more detail later, MyoRing implantation (CISIS) differs from ICRS in that no sutures for closing the cornea are required as the opening is not radial and has no weakening effect on the cornea; it has lamellar and biomechanically neutral properties and is therefore self-sealing (as in clear corneal cataract incisions). Moreover, CISIS does not require a circular tunnel into which implants are inserted as in ICRS. All it takes is a corneal pocket to keep the system biomechanically neutral. This helps to avoid complications typical of ICRS, such as extrusions and corneal melting. The corneal pocket in CISIS has no spatial expansion; it is a virtual gap without a volume which is located between the anterior and posterior lamella of the cornea, even invisible under the biomicroscope. It resembles a laser-assisted in situ keratomileusis (LASIK) flap, which has no volume between the anterior and posterior lamella of the cornea either. The difference between the corneal pocket in CISIS and the flap in LASIK lies in the fact that LASIK weakens the cornea through the wide opening of the lamellar cut of the cornea, whereas the corneal pocket in CISIS is virtually fully closed and therefore biomechanically neutral.

Three months following CISIS/MyoRing implantation, the patient had an uncorrected vision of 0.7 in the left eye that had undergone surgery, and his visual acuity has remained basically unchanged until the present day, 2 years after the intervention:

OS CDVA 0.9 with $+1.0 \mathrm{~s}-1.5 \mathrm{c} \times 175^{\circ}$, UCVA 0.7

The other parameters are SIM K1 $=41.3$, SIM K2 = 44.7 and a corneal depth of $440 \mu \mathrm{m}$. The topography of the treated eye measured during the most recent exam is shown in Figure 2. The clinical findings of the untreated right eye had remained unchanged since the first consultation.

\section{DISCUSSION}

Keratoconus is a disease of the cornea which is characterised by two properties: ${ }^{3}$

1. Impaired vision.

2. Progression.

The normal cornea consists of some 200 regularly stacked collagen lamellae, representing a crucial component of the optical function of the eye. ${ }^{4,5}$ The optical function of the cornea is based on its transparency and its refractive power (Diopters). The transparency of the cornea results from a short-range ordered arrangement of collagen fibrils within the collagen lamellae, similar to a liquid crystal. ${ }^{6}$ The refractive power of the cornea stems from its geometry: the refractive power is indirectly proportional to the central radius of the cornea. ${ }^{7}$ In a normal eye, over 40 Diopters - two thirds of the total refractive power of the eye, which amounts to more than 60 Diopters - are found on the corneal surface. This explains, why even subtle changes in the corneal geometry can lead to considerable vision impairment. As long as the corneal surface has a largely regular curvature, a refraction anomaly (shortsightedness, farsightedness) can still be corrected by eyeglasses. If the corneal surface has an irregular curvature, like in keratoconus, vision impairment can no longer be compensated by eyeglasses, especially



Fig. 2: Sagittal corneal topography measured during the most recent eye exam ( 2 years after MyoRing implantation and 2.5 years after ring segment removal) 
so with higher degrees of irregularity. Pathological changes in the cornea and the loss of vision in patients with keratoconus are associated with the loss of the regular (orthogonal) layering of the collagen lamellae in the corneal stroma. ${ }^{8}$ When a certain degree of irregularity of the corneal geometry is exceeded and vision impairment can no longer be corrected with eyeglasses, the classical approach is to use hard contact lenses. With this method, irregularities on the corneal surface are optically compensated by the tear film underneath the contact lens; the decreased optical function of the cornea is largely replaced by the optical quality of the contact lens or contact lens surface. But because keratoconus has a tendency to progress and the cornea becomes thinner and takes on an increasingly irregular and cone-like shape, there comes a point where even a contact lens will lose its stability on the cornea. According to the classical interpretation, this is the point where corneal transplantation (keratoplasty) is commonly performed. The disadvantage is that the optical function of the eye often varies considerably after keratoplasty and the outcome is often unsatisfactory. ${ }^{9-11}$ Furthermore, it needs to be noted that keratoplasty, and the large number of sickness leaves, work absences and poor work performance associated with it, are a huge financial burden on the economy.

Here is where the benefit of special corneal implants like ring segments (ICRS) and MyoRing CISIS comes in. The aim is to 'bridge' the gap between contact lenses and keratoplasty by a minimally invasive intervention, or in other words, to largely replace contact lenses and keratoplasty by an effective, safe and minimally invasive intervention.

Ring segments (ICRS) were originally developed and used for the treatment of mild shortsightedness of up to -4 Diopters. ${ }^{12-14}$ Because of the relatively poor predictability of the results, they were soon replaced by the concurrently developed methods using excimer laser, such as photorefractive keratectomy (PRK) and LASIK. ${ }^{15-17}$ It was not before the early years of the millennium that ICRS was gradually applied as a method for the treatment of keratoconus. ${ }^{18}$

The basic mechanism of action of ICRS is based on a shortening of the central arc length of the cornea by adding volume to its peripheral parts (Fig. 3).

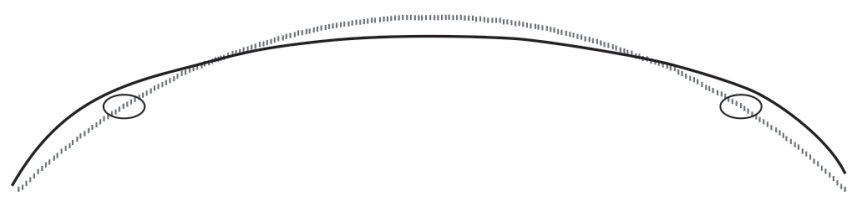

Fig. 3: Schematic representation of the shortening of the arc length by adding peripheral volume to the cornea
When peripheral volume is added, the collagen lamellae have to take a 'detour' around the volume (implant). This 'detour' reduces the central arc length of the cornea, thus flattening the central part and changing the refractive power of the cornea.

In the case of keratoconus, ICRS also serves to regularize the corneal shape to a certain extent, which may lead to postoperative vision improvement. ${ }^{18}$

A novel approach to keratoconus treatment is CISIS, a method which is based on a new technology. ${ }^{19}$

This new method differs from ICRS in that it does not use radial keratotomy to produce a circular tunnel, into which the ring segments are inserted. In CISIS, a PocketMaker ultrakeratome is used to create a lamellar cut in the corneal interior (corneal pocket), which is basically invisible from the outside and represents a virtual gap of $9 \mathrm{~mm}$ in diameter and $300 \mu \mathrm{m}$ in depth. Through a 4 $\mathrm{mm}$ wide temporal lamellar access, the MyoRing is implanted into the corneal pocket. To master this challenge, the MyoRing is compressed by special forceps prior to being implanted, so that it fits through the narrow access and can be inserted into the corneal pocket. The MyoRing combines two apparently contradictory properties: It is rigid enough to stabilize the geometry of the target cornea and also flexible enough (shape memory) to be inserted through a tiny opening in the cornea. After being implanted, the MyoRing readopts its original circular shape. The PocketMaker ultrakeratome is extremely accurate and suited for safely and precisely cutting an appropriate pocket-large enough to accommodate a MyoRing implant of $8 \mathrm{~mm}$ in diameter - even into corneal tissue with heavily reduced thickness (merely $350 \mu \mathrm{m}$ ), such as found in patients with highly advanced keratoconus. In cases, where the corneal thickness is reduced to below $350 \mu \mathrm{m}$, keratoplasty remains the only available option.

The MyoRing is a full ring implant and can therefore not be implanted into a circular tunnel like ICRS; it needs to be inserted into a corneal pocket. In terms of efficacy and safety, the CISIS/MyoRing technique has significant clinical and biomechanical advantages over ICRS. It is quite common that ring segments prove to be ineffective and need to be replaced by a MyoRing; the MyoRing is occasionally even implanted without withdrawal of the ineffective ring segment. ${ }^{20}$

Those who prefer ICRS over the MyoRing occasionally argue that with ICRS the cornea is only peripherally 'manipulated' along a circular tunnel, with the central part of the cornea remaining unaffected, while CISIS and MyoRing also involve the center of the cornea by cutting a $9 \mathrm{~mm}$ wide corneal pocket into its interior. In reply to this argument it needs to be noted that the LASIK method also creates a flap in the central part of the cornea. The 
lamellar cut through the center of the cornea-in the form of a flap as in LASIK, or in the form of a closed pocket as in CISIS-does not lead to an optical impairment. ${ }^{21,22}$ The difference between the corneal pocket in CISIS and the flap in LASIK is more of a biomechanical nature, as LASIK weakens the cornea through the wide opening of the lamellar cut of the cornea (flap), whereas the lamellar cut in CISIS is virtually fully closed (pocket) and therefore biomechanically neutral (Fig. 4).

Since the corneal flap, which was broadly detached from the cornea, actually never heals, the remaining biomechanically relevant corneal thickness $d$ is far less than the anatomical thickness and the cornea is weakened by the thickness of the flap plus the ablated tissue in LASIK. ${ }^{23,24}$

With CISIS, this is entirely different. Since the lamellar cut in CISIS (pocket) is not widely opened, the biomechanical properties are not compromised and the biomechanically relevant corneal thickness is the same as the anatomical thickness, as shown in Figure 5. 24,25

From an anatomical and biomechanical viewpoint, the corneal pocket in CISIS is therefore an 'inversed' flap, as shown in Figure 6. What in LASIK is the large opening of the lamellar cut in the form of a flap that is merely connected to the corneal tissue via a thin hinge, which basically excludes the possibility of a transmission of forces between the anterior corneal lamella and the remaining cornea, can be equated to a narrow opening in CISIS, which provides access to the lamellar cut (pocket) which is otherwise fully closed around the entire

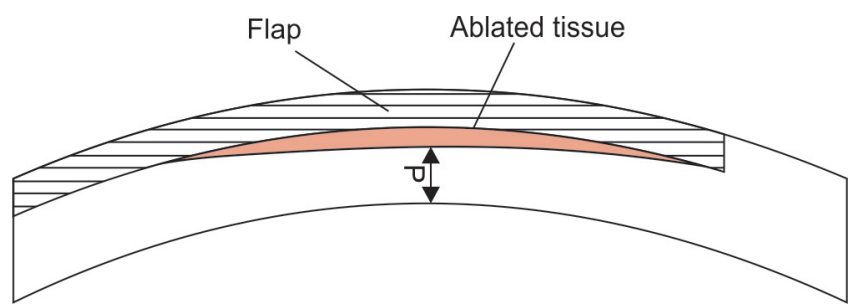

Fig. 4: Schematic representation of the corneal cross section following LASIK. The dashed lines indicate the corneal flap, which was broadly opened (vertical cut into the corneal surface), and the tissue ablated by the excimer laser (black) (d-biomechanically (remaining) relevant thicknes of the cornea)

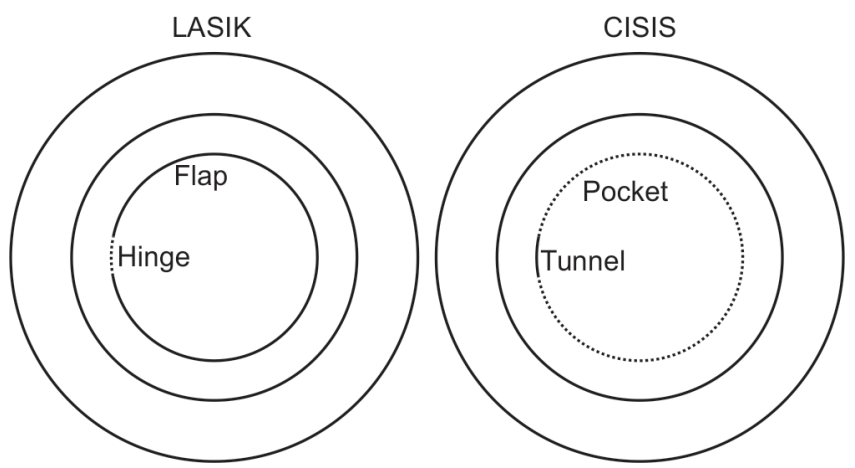

Fig. 6: Comparison of biomechanical properties of LASIK and CISIS circumference. Corneal intrastromal implantation system therefore differs from LASIK in that the transmission of forces between the anterior lamella and the remaining cornea is unimpaired. ${ }^{23-25}$

When some ophthalmologists erroneously equate the biomechanical relevance of a flap in LASIK to that of a corneal pocket in CISIS, their misconception is not of a physical or medical nature but the result of insufficient knowledge of the laws of biomechanics. The depth or extension of a lamellar cut in the cornea is of no biomechanical or clinical relevance, as long as it is generally closed around the entire circumference and not in contact with the corneal surface, as is the case with the corneal pocket. ${ }^{23-25}$ The transcorneal pressure resulting from the difference between intraocular pressure and atmospheric pressure is converted into a tension that acts parallel to the cornea (Fig. 7). ${ }^{24}$ This is also the underlying reason why the collagen fibers of the cornea are largely arranged in orthogonal layers (lamellae): the tissue is optimally adjusted to the distribution of forces. ${ }^{8,24}$

A circular tunnel as in ICRS is neither optically nor biomechanically advantageous when compared to a corneal pocket in CISIS.

Contrarily, the insertion of the ring segments into the circular tunnel produces a biomechanical imbalance, which is not only the cause for complications typically associated with ICRS, such as extrusion and melting of corneal tissue, but also the explanation why ring segments are less efficient than the MyoRing (Fig. 8).

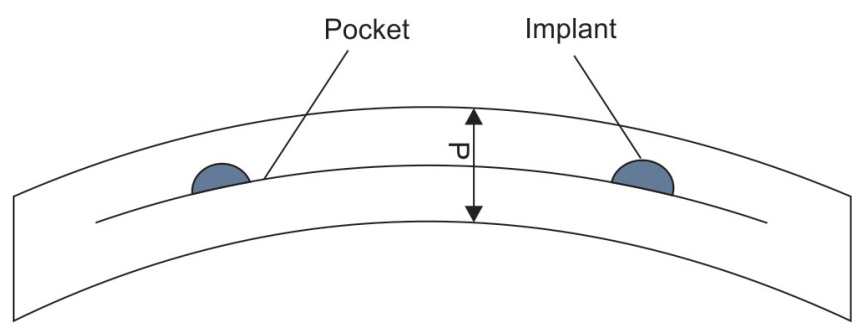

Fig. 5: [The CISIS method creates a practically closed corneal pocket (d-biomechanically (remaining) relevant thickness of the cornea)]

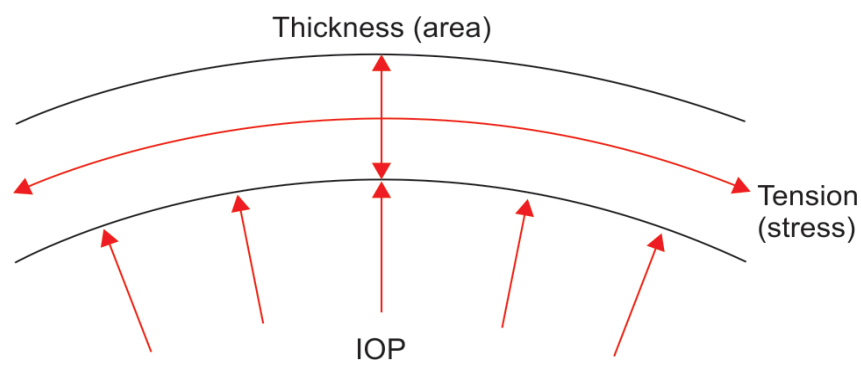

Stress $=$ force (IOP) area (thickness)

tensile strength $=$ maximum stress possible

Fig. 7: Distribution of forces within the cornea 


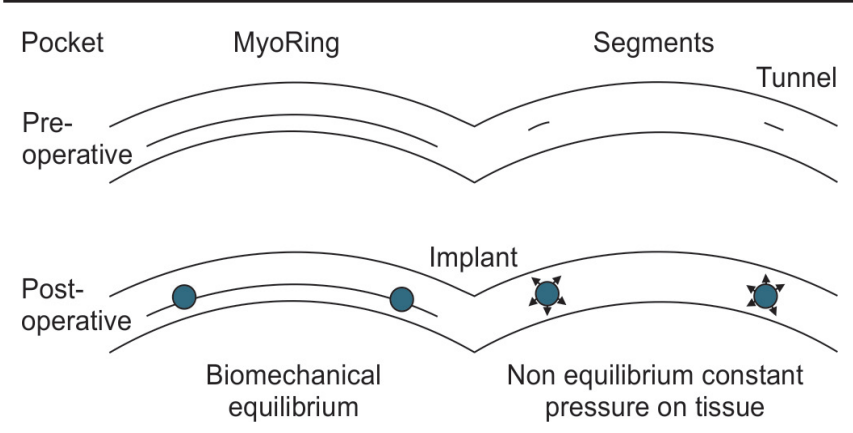

Fig. 8: Comparison of biomechanical conditions in CISIS (MyoRing) and ICRS (ring segment) treatment

Since, the MyoRing is inserted into a virtually fully closed corneal pocket of $9 \mathrm{~mm}$ in diameter, and the MyoRing itself has a maximum diameter of $8 \mathrm{~mm}$, the latter can not cause a build-up of tissue pressure following implantation. The deformation of the cornea around the MyoRing takes place until the biomechanical equilibrium of the cornea-MyoRing system is newly restored. This is in contrast to ICRS, where the implantation of ring segments into a circular tunnel leads to a local build-up of pressure in the surrounding tissue, potentially resulting in pressure atrophies and consequently in clinical symptoms like extrusion and melting of corneal tissue located on top of the implant. ${ }^{26}$ In keratoconus, this is all the more significant as ring segments have typical endings, which the MyoRing does not have. These endings are exposed to additional forces, resulting from torque resistance produced by the irregular geometry of the cornea, and which are transmitted to the anterior corneal lamella. This further enhances the risk of melting of corneal tissue induced by pressure atrophy. Complications, such as implant extrusion and melting of corneal tissue, which are typical symptoms in ICRS, are practically non-existent in MyoRing implantation. ${ }^{22,27}$ This makes CISIS a much safer keratoconus treatment method than ICRS, and also much more efficient in all stages of the disease. ${ }^{22,27-31}$

Another important factor for the much higher efficacy of CISIS over ICRS is that the MyoRing can be centered by using the true postoperative optical axis, which allows the surgeon to use all three potential degrees of freedom (diameter, thickness and position of implant) to achieve an optimal result, whereas in ICRS he has only one degree of freedom (implant thickness) available for an optimised outcome. ${ }^{25}$ Therefore, CISIS always allows to achieve the best result in every given case. In the case of keratoconus, with its corneal irregularity, the individual patient can determine the optical axis (the 'point of vision' through the cornea), i.e. the fixation, so as to achieve the best possible vision. If the corneal geometry changes, the optical axis and the optimal point of fixation also changes. In ICRS implantation, the position of the ring segments is determined by using the preoperative geometry of the cornea as a reference; the circular tunnel is adequately positioned to correspond to the preoperative fixation. The position of the ring segments is then determined by the position of the circular tunnel. By inserting the ring segments into the circular tunnel, however, the corneal geometry, the fixation and the optimal point of vision through the cornea change. There is neither a theoretical nor a practical possibility to assess the situation after the surgical intervention preoperatively. Yet it is of crucial importance for the outcome as well as for the visual acuity to be achieved that the implants are optimally positioned to suit real postoperative conditions. When the centering is optimised by merely $0.5 \mathrm{~mm}$, this may lead to significant improvements in vision (Fig. 9). ${ }^{25}$

The drawback of ICRS is that the postoperative position of the implants is bound by the preoperative

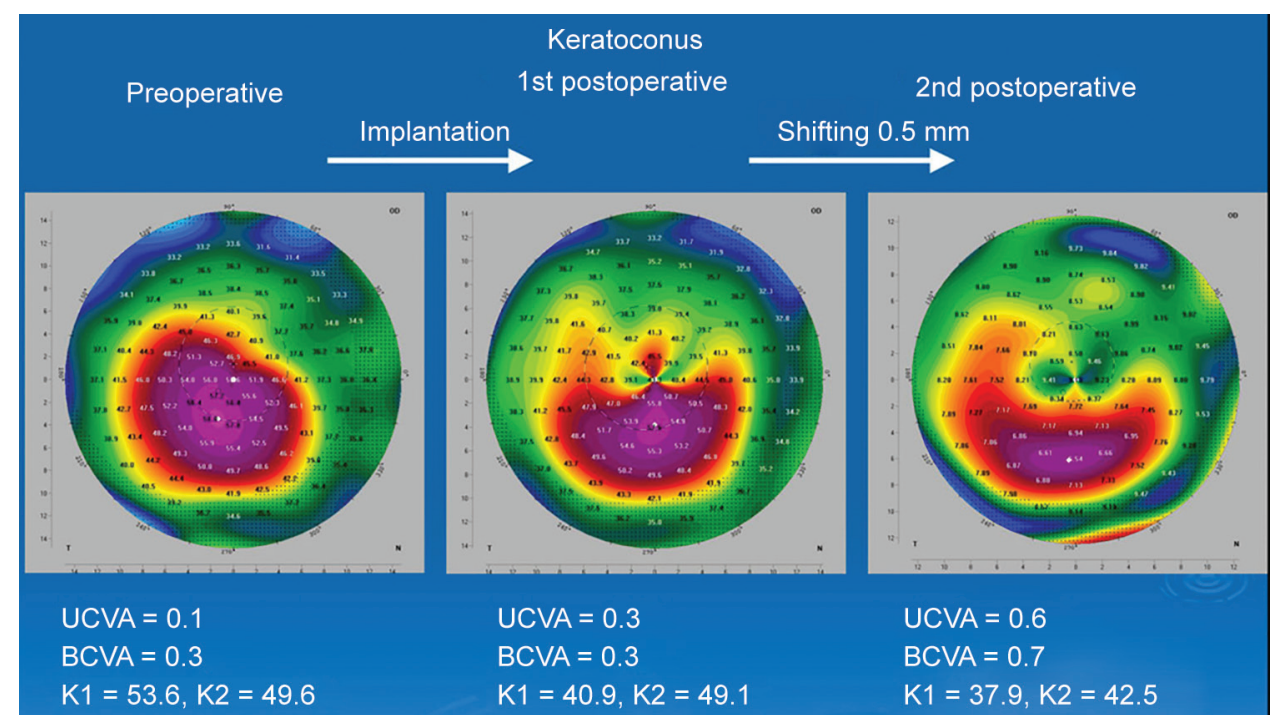

Fig. 9: Optimizing the position by merely $0.5 \mathrm{~mm}$ may lead to a significantly improved outcome 
MyoRing Treatment of Keratoconus

conditions, which implies that an optimized vision for the individual patient can not possibly be achieved. With the MyoRing implantation, this is entirely different. The MyoRing (diameter: $5-8 \mathrm{~mm}$ ) is implanted into a corneal pocket (diameter: $9 \mathrm{~mm}$ ), where it can be optimally positioned within the corneal pocket during the implantation process by adequate forceps to match the true postoperative optical axis and fixation. The more advanced the keratoconus to be treated the smaller the implant diameter and the more important is the right and optimized position. The patient fixates on a specific fixation point by the use of a suitable surgical microscope, and the surgeon shifts the implant accordingly to assure that the position of the MyoRing inside the cornea is optimized to match the patient's true postoperative fixation and the exact point of vision through the cornea. This optimization process can be performed at the onset of treatment or at any other point of time after the intervention, because the corneal pocket can be easily opened to allow access to the implant and optimize its position anytime. ${ }^{25}$ The type of keratoconus-centrally or decentrally located keratoconus-does not matter. ${ }^{32}$ The reason is the mechanism of action, in which CISIS differs considerably from ICRS. Physically, CISIS draws on the mathematical branch of topology, according to which any point on a 'soft surface' that is tightened to a closed rigid structure is determined by the circumferential shape of the rigid structure. ${ }^{32,33}$ The drum is a good example: if we tighten the soft drum surface (skin) to the edge of a pipe-shaped structure, we get a drum. Each point of the drum surface is determined by the geometry of the pipe end to which the drum surface is fastened. If the pipe end is smooth and aligned perpendicular to the pipe axis, we get an optimally flat and regular drum surface. The cornea and the MyoRing behave analogously. Hence, a closed rigid ring with a consistent thickness around its circumference is perfectly suited to regularize the cornea in its optical center. Figure 10 shows that in CISIS the surgeon needs to know nothing about the type of keratoconus. It is basically unimportant how the cornea was warped prior to the surgical intervention; the even ring is forced onto the cornea and regularizes the latter. ${ }^{32}$

This is not the case with the ring segments, which are not closed and more or less 'float' inside the cornea. This is why they only serve to balance out local inhomogeneities and have complicated nomograms to take account of as many corneal geometries as possible.

What has been said so far highlights the main aspects of visual rehabilitation in keratoconus patients. But we also need to look at the second property of the disease, which is its progression. Some progress has been achieved in recent years by a new method called corneal cross-linking (CXL), which helps to preserve the patient's
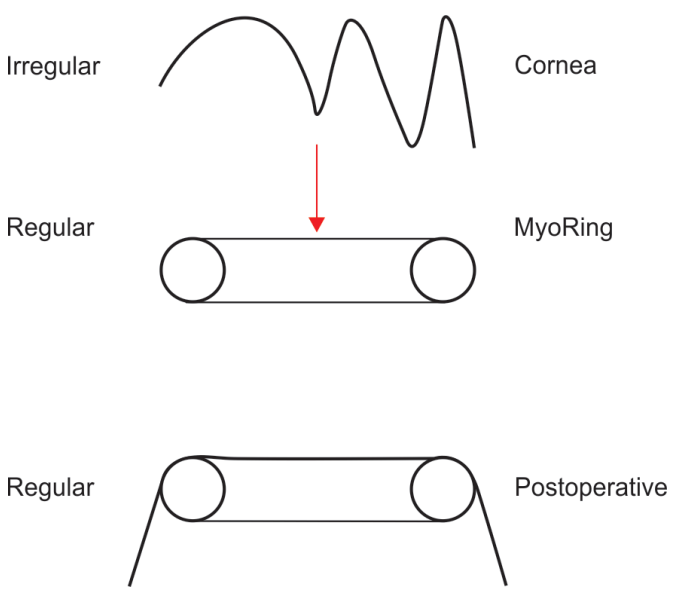

Fig. 10: Topological mechanism of action of the MyoRing

visual acuity in the early stages of keratoconus. ${ }^{34,35}$ This method consists in removing the corneal epithelium, thus exposing the Bowman's layer, and applying riboflavin drops to the eye for half an hour. The cornea, which is now saturated with riboflavin, is subjected to UV-A light with a dose parameter of $5,4 \mathrm{~J} / \mathrm{cm}^{2}$. Since the corneal epithelium is removed, this treatment may cause the patient considerable pain for up to 3 days following the intervention. Another option is to combine ICRS and CXL in the treatment of keratoconus. ${ }^{36}$ Corneal intrastromal implantation system may be combined with CXL, too; but because of the corneal pocket, rather than applying riboflavin drops to the cornea now deprived of its epithelium as in classical CXL, riboflavin can be flushed into the corneal pocket instead. ${ }^{37,38}$ This allows to effectively perform MyoRing implantation and CXL without epithelial debridement during one single session while avoiding postoperative pain. Long-term observations seem to indicate that after MyoRing implantation, even when not combined with CXL, there is no progression of the disease. Many cases even report an increase in corneal thickness after the intervention. ${ }^{27,39,40}$ As biomechanical analyses demonstrate, this has something to do with the special closed ring structure of the MyoRing. ${ }^{41}$ The collagen lamellae of the cornea are orthogonally arranged toward the peripheral cornea ${ }^{8}$ and change their direction into a circular pattern at the corneal limbus. ${ }^{42}$ This endows the limbus with a much higher elastic modulus and a higher biomechanical strength than the central cornea. ${ }^{24,43}$ From a biomechanical perspective, an implanted MyoRing acts like a second limbus within the cornea, dividing the forces that act on the cornea into two areas, ${ }^{41}$ similar to a beam that supports the ceiling of a residential unit. The beam also distributes the load imposed on the ceiling onto two areas, basically splitting the load per area in half. A half-beam or an incomplete beam is, of course, unable to distribute this load. This may be compared to 
the ring segments, which cannot halt the progression of the disease. ${ }^{41,44,45}$ The force needed to separate the segments or separate the ends of incomplete rings is close to nil, while the high elastic modulus of the MyoRing material—due to the closed structure-can be fully utilised to achieve biomechanical strength. A MyoRing implant can therefore increase the strength of a cornea, depending on its size, by a factor of 2 to 3 , which means that a cornea with an anatomical thickness of $400 \mu \mathrm{m}$ displays the biomechanical properties of a cornea that is between 800 and $1200 \mu \mathrm{m}$ thick. ${ }^{24,41}$ It is therefore possible, even in suspicious cases of myopia where laser treatment for vision correction is not an option, to combine MyoRing implantation with laser therapy to maximally extend the optical zone. ${ }^{46}$

\section{REFERENCES}

1. Rabinowitz YS. Keratoconus. Surv Ophthalmol 1998;42: 297-319.

2. Ziaei $\mathrm{H}$, et al. Epidemiology of keratoconus in an Iranian population. Cornea 2012;31:1044-1047.

3. Krachmer JH, Feder RS, Belin MW. Keratoconus and related noninflammatory corneal thinning disorders. Surv Ophthalmol 1984;28:293-322.

4. Komai Y, Ushiki T. The three-dimensional organisation of collagen fibrils in the human cornea and sclera. Invest Ophthalmol Vis Sci 1991;32:2244-2258.

5. Daxer A, et al. Collagen fibrils in the human corneal stroma: structure and ageing. Invest Ophthalmol Vis Sci 1998;39: 644-647.

6. Fratzl P, Daxer A. Structural transformation of collagen fibrils in the corneal stroma during drying. An X-ray scattering study. Biophys J 1993;64:1210-1214.

7. Rabbetts RB. The eye's optical system. In Bennett and Rabbett's Clinical Visual Optics. Butterworth, Heinemann, Elsevier Philadelphia. 4th ed. 2007.

8. Daxer A, Fratzl P. Collagen fibril orientation in the human corneal stroma and its implications in keratoconus. Invest Ophthalmol Vis Sci 1997;38:121-129.

9. Kelly TL, Coster DJ, Williams KA. Repeat penetrating corneal transplantation in patients with keratoconus. Ophthalmology 2011;118:1538-1542.

10. Rodriguez LA, et al. Penetrating keratoplasty versus intrastromal corneal ring segments to correct bilateral corneal ectasia: preliminary study. J Cataract Rafrect Surg 2007;33:488-496.

11. Bahar I, et al. Comparison of three different techniques of corneal transplantation for keratoconus. Am J Ophthalmol 2008;146:905-912.

12. Blavatskaya ED. Intralamellar homoplasty for the purpose of relaxation of refraction of the eye. Arch Soc Ophthalmol Optom 1968;6:311-325 (translated from Oftalmol Zh 1966; 7:530-537.

13. Nose W, Neves RA, Schanzlin DJ, Belfort R. Intrastromal corneal ring-one year results of first implants in humans: a preliminary nonfunctional eye study. Refract Corneal Surg 1993;9:452-458.

14. Ruckhofer J, Stoiber J, Twa MD, Grabner G. Correction of astigmatism with short arc-length intrastromal corneal ring segments: preliminary results. Ophthalmol 2004;110: 516-524.

15. Trokel SL, Srinivasan R, Braren B. Excimer laser surgery of the cornea. Am J Ophthalmol 1983;96:710-715.

16. Seiler T, Berlin MS, Bende T, Trokel S. Excimer laser keratectomy for correction of astigmatism. Am J Ophthalmol 1988;105:117-120.

17. Speicher L, Daxer A, Göttinger W. Laserchirurgische Korrektion der Fehlsichtigkeit. Erfahrungen und bisherige Ergebnisse. Ber. nat.-med. Verein Innsbruck 1995;82:335-345.

18. Colin, et al. Correcting keratoconus with intracorneal rings. J Cataract Refract Surg 2000;26:1117-1122.

19. Available at: www.dioptex.com

20. Behrouz MJ, et al. Intacs followed by MyoRing implantation in severe keratoconus. J Refract Surg 2013;29:364-366.

21. Kato N, et al. Five-year outcome of LASIK for myopia. Ophthalmol 2008;115:839-844.

22. Daxer A, Mahmood H, Venkateswaran RS. Intracorneal continous ring implantation for keratoconus: one year followup. J Cataract Refract Surg 2010;36:1296-1302.

23. Reinstein DZ, Archer TJ, Randleman JB. Mathematical model to compare the relative tensile strength of the cornea after PRK, LASIK, and small incision lenticule extraction. J Refract Surg 2013;29:454-460.

24. Daxer A. Biomechanics of the cornea. Int J Keratoconus and Ectatic Corneal Dis 2014;3:57-62.

25. Daxer A. Adustable intracorneal ring in a corneal pocket for keratoconus. J Refract Surg 2010;26:217-221.

26. Ferrer $\mathrm{C}$, et al. Causes of intrastromal corneal ring segment explantation: clinicopathologic correlation analysis. J Cataract Refract Surg 2010;36:970-977.

27. Jabbarvand M, Salamatrad A, Hashemian H, Khodaparast M. Continous corneal intrastromal ring implantation for treatment in keratoconus in an Iranian population. Am J Ophthalmol 2013;155:837-842.

28. Mahmood H, Venkateswaran RS, Daxer A. Implantation of a complete corneal ring in an intrastromal pocket for keratoconus. J Refract Surg 2011;27:63-68.

29. Alio JL, Shabayek MH, Artola A. Intracorneal ring segments for keratoconus correction: long-term follow-up. J Cataract Refract Surg 2006;32:978-985.

30. Shabayek MH, Alio JL. Intrastromal corneal ring segment implantation by femtosecond laser for keratoconus correction. Ophthalmol 2007;114:1643-1652.

31. Shetty $\mathrm{R}$, et al. Intacs in advanced keratoconus. Cornea 2008;27:1022-1029.

32. Daxer A. MyoRing for central and noncentral keratoconus. Int J Keratoconus and Ectatic Corneal Diseases 2012;1:117-119.

33. Daxer A. Corneal intrastromal implantation surgery for the treatment of moderate and high myopia. J Cataract Refract Surg 2008;34:194-198.

34. Spoerl E, Huhle M, Seiler T. Induction of cross-links in corneal tissue. Exp Eye Res 1998;66:97-103.

35. Vinciguerra P, Albe E, Trazza S, Rosetta P, Vinciguerra R, Seiler T, Epstein D. Refractive, topographic, tomographic, and abberometric analysis of keratoconic eyes undergoing corneal cross-linking. Ophthalmol 2009;116:369-378.

36. Avni-Zauberman N, Rootman DS. Cross-linking and intracorneal ring segments: review of the literature. Eye Contact Lens 2014;40:365-370.

37. Daxer A, Mahmoud H, Venkateswaran RS. Corneal crosslinking and visual rehabilitation in keratoconus in one 
session without epithelial debridement: new technique. Cornea 2010;29:1176-1179.

38. Studeny P, Krizova D, Stranak Z. Clinical outcomes after complete intracorneal ring implantation and corneal crosslinking in an intrastromal pocket in one session for keratoconus. J Ophthalmol 2014;568128 DOI:10.1155/2014/568128.

39. Alio JL, Pinero DP, Daxer A. Clinical outcomes after complete ring implantation in corneal ectasia using the femtosecond laser technology: a pilot study. Ophthalmol 2011;118: 1282-1290.

40. Daxer A. Corneal thickness after MyoRing implantation for keratoconus. Int J Keratoconus and Ectatic Corneal Dis 2014; 3:15-19.

41. Daxer A. Biomechanics of corneal ring implants. Cornea 2015 (In press).
42. Aghamohammadzadeh H, Newton RH, Meek KM. X-ray scattering used to map the preferred collagen orientation in the human cornea and limbus. Structure 2004;12:249-256.

43. Meek KM. The cornea and sclera. In: Fratzl P, editor. Collagen: structure and mechanics. Springer New York, 2010.

44. Alio JL, Vega-Estrada A, Esperanza S, Barraquer RI, Teus MA, Murta J. Intrastromal corneal ring segments: how successful is the surgical treatment of keratoconus? Middle East Afr J Ophthalmol 2014;21:3-9.

45. Chhadva $\mathrm{P}$, et al. Intrastromal corneal ring segment explantation in patients with keratoconus: causes, techniques and outcomes. J Refract Surg 2015;31:392-397.

46. Daxer A. MyoRing treatment for cases of Myopia not eligible for laser vision correction. Int J Keratoconus and Ectatic Corneal Dis 2014;3:20-22. 